TITLE:

\title{
Efficient delivery of immunostimulatory DNA to mouse and human immune cells through the construction of polypod-like structured DNA.
}

\section{$\operatorname{AUTHOR}(\mathrm{S}):$}

Uno, Shota; Nishikawa, Makiya; Mohri, Kohta; Umeki, Yuka; Matsuzaki, Noriyuki; Takahashi, Yuki; Fujita, Haruyuki; Kadowaki, Norimitsu; Takakura, Yoshinobu

\section{CITATION:}

Uno, Shota ... [et al]. Efficient delivery of immunostimulatory DNA to mouse and human immune cells through the construction of polypod-like structured DNA.. Nanomedicine 2014, 10(4): 765-774

\section{ISSUE DATE:}

2014-05

URL:

http://hdl.handle.net/2433/187794

\section{RIGHT:}

C 2014 Elsevier Inc.; この論文は出版社版でありません。引用の際には 出版社版をご確認ご利用ください。; This is not the published version. Please cite only the published version. 


\title{
Efficient delivery of immunostimulatory DNA to mouse and human immune cells through the construction of polypod-like structured DNA
}

\author{
Shota Uno, M.S.," Makiya Nishikawa, Ph.D., ${ }^{* \dagger, 1}$ Kohta Mohri, Ph.D., ${ }^{*}$ Yuka \\ Umeki, B.S.," Noriyuki Matsuzaki, B.S., Yuki Takahashi, Ph.D., ${ }^{* \dagger}$ \\ Haruyuki Fujita, M.D., ${ }^{\ddagger}$ Norimitsu Kadowaki, M.D., ${ }^{\ddagger}$ Yoshinobu Takakura, \\ Ph.D."
}

*Department of Biopharmaceutics and Drug Metabolism, Graduate School of Pharmaceutical Sciences, Kyoto University, Sakyo-ku, Kyoto 606-8501, Japan; ' Institute for Integrated Cell-Material Sciences (iCeMS), Kyoto University, Sakyo-ku, Kyoto 606-8501, Japan; ${ }^{\ddagger}$ Department of Haematology and Oncology, Graduate School of Medicine, Kyoto University, Sakyo-ku, Kyoto 606-8507, Japan.

Short Title: Enhanced activation of immune cells by polypodna

${ }^{1}$ Corresponding author: Makiya Nishikawa, Ph. D., Department of Biopharmaceutics and Drug Metabolism, Graduate School of Pharmaceutical Sciences, Kyoto University, Sakyo-ku, Kyoto 606-8501, Japan. Phone: +81-75-753-4580; Fax: +81-75-753-4614. E-mail: makiya@pharm.kyoto-u.ac.jp

Word count for abstract: 144

Complete manuscript word count (body text and figure legends): 4,880

Number of references: 43

Number of figures/tables: $6 / 2$

This work is supported in part by a Grant-in-Aid for Scientific Research (B) (23390010) from the Japan Society for the Promotion of Science, by a Grant-in-Aid for Scientific Research on Innovative Areas "Carcinogenic spiral” (25114706) from Ministry of Education, Culture, Sports, Science and Technology of Japan, and by a Cross-Disciplinary Collaborative Research Promotion Project from Institute for Integrated Cell-Material Sciences, Kyoto University. The authors have no conflict of interest and no disclosures. 


\begin{abstract}
Investigation of mouse macrophage-like RAW264.7 cells showed that the immunostimulatory activity of CpG DNA is increased by formation of polypod-like structured DNA (polypodna), an assembly consisting of three or more oligodeoxynucleotides. To apply $\mathrm{CpG}$ polypodna to immunotherapy, its activity was examined in murine dendritic DC2.4 cells, splenic macrophages, and bone marrow-derived dendritic cells (BMDCs). In all cell types, increasing the pod number increased the cellular uptake of DNA and cytokine release. No significant release of cytokines was observed in macrophages lacking Toll-like receptor 9. Similar results were obtained after intradermal injection of polypodna. The polypodna preparations produced significantly higher amounts of interferon $\alpha$ in human peripheral blood mononuclear cells (PBMCs) compared with single-stranded DNA. The conditioned medium of hexapodna-treated human PBMCs effectively inhibited activity of a hepatitis $\mathrm{C}$ virus subgenomic replicon reporter system. These results indicate that polypodna preparations are useful as an immunostimulator.
\end{abstract}

Keywords: CpG DNA; Toll-like receptor 9; dendritic cell; peripheral blood mononuclear cell 


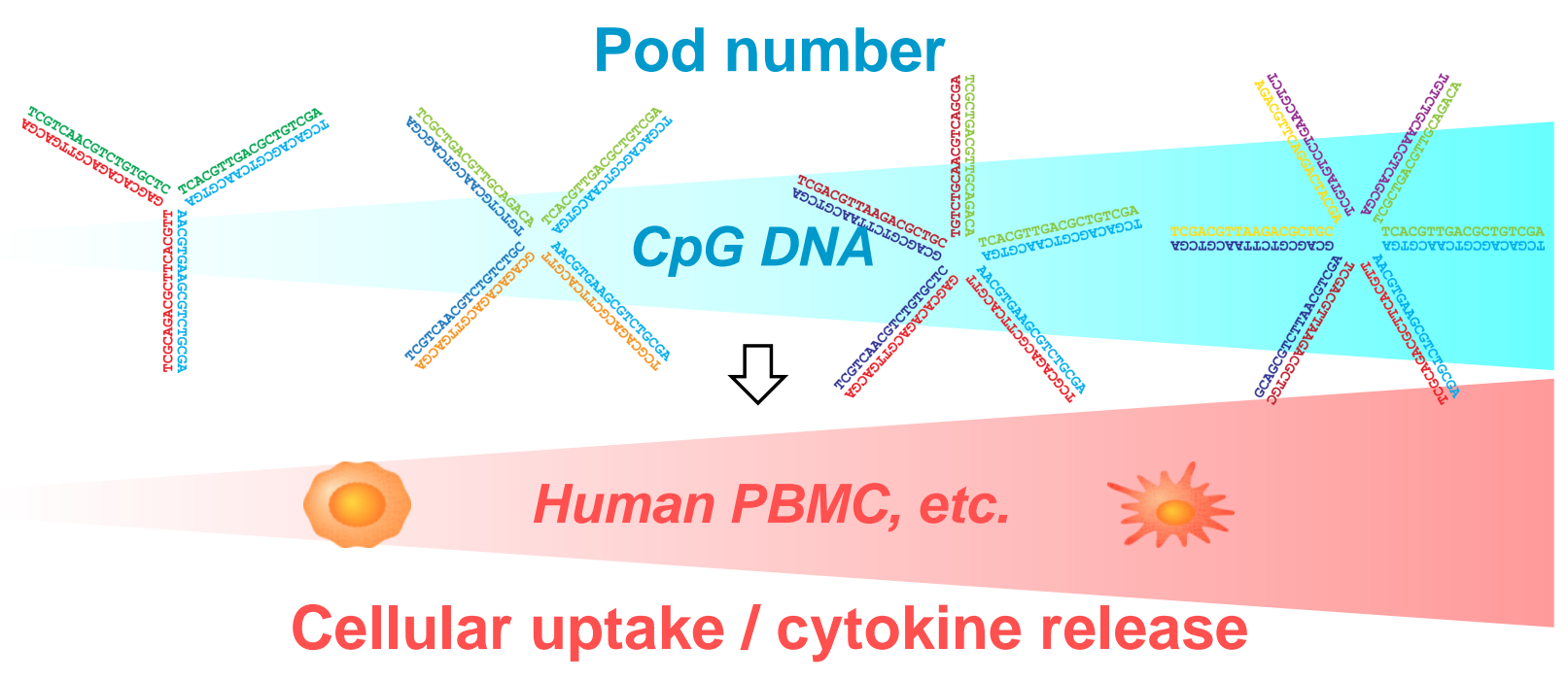




\section{Introduction}

Stimulation of innate immune cells leads to secretion of proinflammatory cytokines, which, in turn, causes inflammation. Such inflammation is likely essential for the induction of efficient immune responses to a variety of pathogens. ${ }^{1}$ Ligands for Toll-like receptors (TLRs) have therapeutic potential, because TLR ligation initiates a signaling pathway that activates nuclear factor $\kappa \mathrm{B}$, which is a master transcription factor controlling the expression of proinflammatory cytokines.

One of the most-studied TLR ligands is DNA containing unmethylated cytosine-phosphate-guanine dinucleotides (CpG motifs), the ligand for TLR9. ${ }^{2,3}$ This specific DNA is abundant in viral, bacterial and mitochondrial DNA, and it is categorized as a pathogen- or damage-associated molecular pattern. ${ }^{4}$ TLR9 ligation induces the release of cytokines such as tumor necrosis factor (TNF)- $\alpha$, interleukin (IL)-12 and interferon (IFN)- $\alpha .^{5-8}$ Although natural phosphodiester CpG DNA is a ligand for TLR9, its susceptibility to enzymatic degradation limits its therapeutic applications. Therefore, phosphorothioate-stabilized CpG DNA has been used to induce cytokine production. ${ }^{9,10}$ This stabilized DNA, however, is associated with adverse reactions, such as renal damage. ${ }^{11}$ Therefore, other approaches for increasing the activity of CpG DNA are required to enhance its therapeutic potency.

Recent advances in DNA nanotechnology have resulted in the development of uniquely structured DNA assemblies. CpG DNA has been linked with or incorporated into these assembled DNA systems, such as Y- or X-shaped DNA, ${ }^{12,13}$ dendrimer-like DNA, ${ }^{14}$ DNA tetrahedra ${ }^{15}$ and DNA origami. ${ }^{16}$ We have recently developed a series of polypod-like structured DNAs, or polypodna, which is a DNA assembly consisting of three or more oligodeoxynucleotides (ODNs), and found that the immunostimulatory activity of CpG DNA is a function of the structural characteristics of the polypodna. For example, both pod number 
and size are important parameters that determine the cellular uptake of polypodna preparations in mouse macrophage-like RAW264.7 cells and the following cytokine production. $^{17}$

Despite these advances, additional studies of CpG DNA-containing polypodna in cells other than RAW264.7 are required, since the cellular response to CpG DNA is highly cell type- and species-specific. ${ }^{18,19}$ Human macrophages do not express TLR9, ${ }^{20}$ and the major human cells responding to CpG DNA are considered to be plasmacytoid dendritic cells. ${ }^{21}$ In addition, we have demonstrated that primary macrophages respond differently to CpG DNA than RAW264.7 cells. ${ }^{19}$

Based on these considerations, this study was designed to examine the immunostimulatory activity of polypodna in murine immune cells that play important roles in the response to CpG DNA. We also examined the immunostimulatory activity of polypodna in vivo after intradermal injection into mice. Finally, human peripheral blood mononuclear cells (PBMCs) were also used to investigate whether polypodna preparations effectively activate human immune cells. 


\section{Methods}

\section{Chemicals}

Roswell Park Memorial Institute (RPMI) medium and Dulbecco's modified Eagle's medium (DMEM) powder were obtained from Nissui Pharmaceutical, Co., Ltd. (Tokyo, Japan). DMEM (liquid) was purchased from Life Technologies (Carlsbad, CA) and used only for LucNeo\#2 cells. Opti-modified Eagle's medium (Opti-MEM) and Iscove's modified Dulbecco's medium (IMDM) and MEM non-essential amino acids (NEAA) were purchased from Life Technologies. Fetal bovine serum (FBS) was obtained from Biowest (Nuaille, France), monothioglycerol and cytochalasin B were obtained from Wako Pure Chemical (Osaka, Japan), G418 was purchased from Nacalai Tesque (Kyoto, Japan), and chloroquine diphosphate salt, dansylcadaverine and lipopolysaccharide (LPS; L3014) were purchased from Sigma-Aldrich (St. Louis, MO).

\section{Animals}

Male C57BL/6 mice were purchased from Japan SLC, Inc (Shizuoka, Japan). TLR9 knockout $\left(\mathrm{TLR}^{-/-}\right)$mice with a C57BL/6 genetic background were purchased from Oriental Yeast Company (Tokyo, Japan). All animal experiments were conducted in accordance with the principles and procedures outlined in the National Institutes of Health Guide for the Care and Use of Laboratory Animals. The protocols for animal experiments were approved by the Animal Experimentation Committee of the Graduate School of Pharmaceutical Sciences, Kyoto University.

\section{Cell culture}

The murine macrophage-like cell line RAW264.7 was cultured in RPMI medium supplemented with $10 \%$ heat-inactivated FBS, $0.2 \%$ sodium bicarbonate, $100 \mathrm{IU} / \mathrm{mL}$ 
penicillin, $100 \mu \mathrm{g} / \mathrm{mL}$ streptomycin and $2 \mathrm{mM}$ L-glutamine. The murine dendritic cell line DC2.4 (kindly provided by Dr. K. L. Rock, University of Massachusetts Medical School, Worcester, MA) was cultured in RPMI medium supplemented with $10 \%$ heat-inactivated FBS, $0.2 \%$ sodium bicarbonate, $100 \mathrm{IU} / \mathrm{mL}$ penicillin, $100 \mu \mathrm{g} / \mathrm{mL}$ streptomycin, $2 \mathrm{mM}$ L-glutamine, $0.5 \mathrm{mM}$ monothioglycerol and 0.1 mM NEAA. These cells were replated on 24-well, 48-well or 96 -well culture plates at a density of $2.5 \times 10^{5}$ cells/well, $1 \times 10^{5}$ cells/well or $5 \times 10^{4}$ cells/well, respectively, and cultured for $24 \mathrm{~h}$ prior to use. LucNeo\#2, a Huh-7 cell harboring self-replicating subgenomic hepatitis $\mathrm{C}$ virus (HCV) RNA replicons with a luciferase reporter, ${ }^{22}$ was kindly provided by Drs. Hijikata and Shimotohno of Institute for Virus Research, Kyoto University, Japan. LucNeo\#2 cells were cultured in DMEM supplemented with $10 \%$ FBS, $2 \mathrm{mM}$ L-glutamine and $0.1 \mathrm{mM}$ NEAA in the presence of $500 \mu \mathrm{g} / \mathrm{mL} \mathrm{G} 418$.

\section{Preparation of mouse bone marrow-derived dendritic cells}

Bone marrow-derived dendritic cells (BMDCs) were generated from 8- to 10-week old male C57BL/6 mice as previously reported. ${ }^{23}$ Briefly, bone marrow cells were isolated by flushing femurs and tibiae with RPMI using a 26-gauge needle. After filtration through a $40 \mu \mathrm{m}$ cell strainer (BD Falcon, Franklin Lakes, NJ), bone marrow cells were resuspended in $0.86 \%$ ammonium chloride for 5 min to lyse the red blood cells. Then, the remaining cells were cultured for 8 days at a density of $5 \times 10^{6}$ cells/mL in RPMI supplemented with $10 \%$ heat-inactivated FBS, $0.2 \%$ sodium bicarbonate, $100 \mathrm{IU} / \mathrm{mL}$ penicillin, $100 \mu \mathrm{g} / \mathrm{mL}$ streptomycin, $2 \mathrm{mM}$ L-glutamine, $0.5 \mathrm{mM}$ monothioglycerol, and $100 \mathrm{ng} / \mathrm{mL}$ mouse recombinant Flt-3 ligand (Peprotech, Rocky Hill, NJ). Cells were grown in humidified air containing $5 \% \mathrm{CO}_{2}$ at $37^{\circ} \mathrm{C}$. On day 7 of culture, non-adherent cells were harvested and used as BMDCs. Cells were replated on 96-well culture plates at a density of $3 \times 10^{5}$ cells/well for the cytokine release experiment. 


\section{Isolation of mouse splenic macrophages}

Spleens were removed aseptically from 8- to 10-week old male C57BL/6 mice, divided with a spatula and filtered through a $40 \mu \mathrm{m}$ cell strainer (BD Falcon) to obtain single-cell suspensions. Red blood cells were lysed by exposure to $0.86 \%$ ammonium chloride for 5 min. After washing three times with phosphate buffered saline (PBS), splenocytes were suspended in RPMI medium supplemented with $10 \%$ FBS, penicillin G (100 U/mL), streptomycin (100 $\mathrm{mg} / \mathrm{mL}$ ), and $0.5 \mathrm{mM}$ monothioglycerol, and plated in $10-\mathrm{cm}$ culture dishes. After a $3 \mathrm{~h}$ incubation in humidified air containing $5 \% \mathrm{CO}_{2}$ at $37^{\circ} \mathrm{C}$, adherent macrophages were washed twice with RPMI-1640 medium to remove non-adherent cells and replated at a density of $4 \times 10^{5}$ cells/well on 48 -well or $96-$ well culture plates.

\section{Isolation of human peripheral blood mononuclear cells}

All experiments using human cells were approved by the Institutional Review Board at the Graduate School of Medicine, Kyoto University, and following the tenets of the Declaration of Helsinki. Human PBMCs were obtained from healthy donors after obtaining their written informed consent. In brief, PBMCs were isolated from peripheral blood using Ficoll-Plaque PLUS (GE Healthcare Piscataway, NJ) density gradient centrifugation according to the manufacturer's protocol. PBMCs collected were replated on 48-well culture plates at a density of $6 \times 10^{5}$ cells/well in Opti-MEM. There were considerable individual differences in the response of cells to $\mathrm{CpG}$ DNA, and only highly responsive cells were used for experiments.

\section{Oligodeoxynucleotides}

Oligodeoxynucleotides (ODNs) with a length of 36-mer were purchased from Integrated DNA Technologies, Inc (Coralville, IA). The sequences of the ODNs are listed in Tables 1 
and 2. Each ODN was given a name, such as mTri-1, hTri-1 and so on, with 'm' for mouse and ' $h$ ' for human, Tri, Tetra, Pen, Hex for tripodna, tetrapodna, pentapodna and hexapodna, respectively, and the ODN number. The ODNs for CpG polypodna for mouse cells were identical to those used in a previous study. ${ }^{17}$ For cellular uptake studies, mTri-2 labeled with Alexa Fluor-488 at the 5' end was purchased from Japan BioService Co., Ltd. (Saitama, Japan).

\section{Preparation of polypodna}

Polypodna samples were prepared by mixing equimolar amounts of their components as previously reported. ${ }^{17}$ In brief, ODNs dissolved in an annealing buffer (10 mM Tris-HCl, pH 8.0, $1 \mathrm{mM}$ ethylenediaminetatraacetic acid, and $150 \mathrm{mM}$ sodium chloride) were mixed at a final concentration of $0.1 \mathrm{mM}$ for each ODN. The mixtures of ODNs were annealed by heating them to $95^{\circ} \mathrm{C}$ and gently cooling to $4^{\circ} \mathrm{C}$ using a thermal cycler. Then, the concentrations of DNA samples were normalized. For cellular uptake studies, Alexa Fluor 488-labeled mTri-2 was used instead of mTri-2. The products of polypodna preparations were analyzed by $6 \%$ polyacrylamide gel electrophoresis (PAGE) at room temperature at $200 \mathrm{~V}$ for 20 min and stained with ethidium bromide for visualization.

\section{Cytokine release}

Cells were incubated with single-stranded (ss) DNA (mTri-2 for mouse cells or hTri-2 for human PBMCs) or polypodna at the indicated concentrations for $24 \mathrm{~h}$. Then, culture supernatants were collected for enzyme-linked immunosorbent assay (ELISA) and kept at $-80^{\circ} \mathrm{C}$ until use. Appropriate cytokines that are highly released from each type of cell were selected as indicators of cellular stimulation. The levels of mouse IL-6, the p40 subunit of IL-12 (IL-12p40) and tumor necrosis factor (TNF)- $\alpha$ were determined using OptEIA ${ }^{\mathrm{TM}}$ sets 
(Pharmingen, San Diego, CA). The levels of human IFN- $\alpha$ were determined using the IFN- $\alpha$-ELISA module set (Bender Med Systems, Vienna, Austria).

\section{Cellular uptake of polypodna}

DC2. 4 cells $\left(5 \times 10^{4}\right.$ cells/well $)$ or splenic macrophages $\left(4 \times 10^{5}\right.$ cells/well $)$ on $96-$ well culture plates were incubated with $2 \mu \mathrm{g} / \mathrm{mL}$ Alexa Fluor 488-labeled DNA samples for 8 or $4 \mathrm{~h}$, respectively, at $37^{\circ} \mathrm{C}$ or $4^{\circ} \mathrm{C}$. Then, cells were washed twice with $200 \mu \mathrm{L}$ PBS and harvested. Finally, the fluorescence intensity of the cells was determined by flow cytometry (FACS Calibur, BD Biosciences, Franklin Lakes, NJ) using CellQuest software (version 3.1, BD Biosciences), and the mean fluorescent intensity (MFI) was calculated as an indicator of cellular uptake. To compare the cellular uptake of ssDNA and polypodna preparations, a normalized MFI was calculated by multiplying the MFI obtained with the fraction of Alexa Fluor 488-labeled ODN in samples, because the amount of Alexa Fluor 488-labeled ODN in the DNA samples was not identical.

\section{Effect of inhibitors on cytokine release and cellular uptake in DC2.4 cells}

Chloroquine (an inhibitor of endosomal fusion and acidification), dansylcadaverine (an inhibitor of clathrin-mediated endocytosis), and cytochalasin B (an inhibitor of phagocytosis) were used to examine the mechanisms involved in the cellular uptake of polypodna preparations. Cells were preincubated for 30 min with $6.25 \mu \mathrm{M}$ chloroquine, $25 \mu \mathrm{M}$ dansylcadaverine, or $5 \mu \mathrm{M}$ cytochalasin $\mathrm{B}$, then incubated with $\mathrm{CpG}$ hexapodna or LPS in the presence of each inhibitor for $24 \mathrm{~h}$. LPS, a ligand for Toll-like receptor 4, was used to confirm whether the DC2.4 cells treated with these inhibitors have the ability to release IL-6. IL-6 release and cellular uptake were examined as described above. 


\section{IL-12p40 production in mice}

Under anesthesia with isoflurane, mice were injected with saline with or without ssDNA, $\mathrm{CpG}$ tripodna or $\mathrm{CpG}$ hexapodna into the dorsal skin at a dose of $100 \mu \mathrm{g}$ DNA/mouse. At 1, 3, 6 , and $9 \mathrm{~h}$ after injection, the blood was collected from the tail vein of mice and serum was collected by centrifugation and stored at $-20^{\circ} \mathrm{C}$ until analysis. The levels of IL-12p40 in the serum were determined by ELISA as described above.

\section{Inhibition of HCV replication in an HCV subgenomic replicon system}

LucNeo\#2 cells were used to examine the inhibition of $\mathrm{HCV}$ replication by conditioned media of polypodna-treated human PBMCs, because the luciferase activity of the cells can be used as an indicator of $\mathrm{HCV}$ replication. Human PBMCs were incubated with ssDNA or CpG hexapodna at a concentration of $2 \mu \mathrm{g} \mathrm{DNA} / \mathrm{mL}$ as described above. Then, the supernatant was collected as the conditioned medium. LucNeo\#2 cells were replated at a concentration of $2 \times 10^{4}$ cells/well on 48 -well culture plates and cultured for $24 \mathrm{~h}$ in DMEM supplemented with 10\% FBS, $100 \mathrm{IU} / \mathrm{mL}$ penicillin, $100 \mu \mathrm{g} / \mathrm{mL}$ streptomycin, and $2 \mathrm{mM}$ L-glutamine. Then, the conditioned medium of PBMCs was diluted 20-fold with DMEM and added to LucNeo\#2 cells, and the cells were harvested after a 24-h culture and lysed. HCV replication was estimated by measuring the luciferase activity of the cell lysates in a luminometer Lumat LB 9507 (Berthold Technologies, Bad Wildbad, Germany).

\section{Statistical analysis}

Differences were statistically evaluated by one-way analysis of variance followed by a TukeyKramer multiple comparisons test for multiple comparison or by Student's $t$-test for comparison between two groups. $P$-values of less than 0.05 were considered to be statistically 
significant. 


\section{Results}

Cytokine release and uptake after addition of polypodna to mouse dendritic DC2.4 cells

Figure 1 shows the sequence and planar representation of $\mathrm{CpG}$ polypodna consisting of three, four, five, or six ODNs for mouse cells. Each ODN was identified with different colors. Figure 2A shows the IL-6 concentrations in the culture media of DC2.4 cells after addition of ssDNA or polypodna preparations containing CpG motifs. All polypodna preparations examined, i.e., tri-, tetra-, penta- and hexapodna, induced higher levels of IL-6 than ssDNA, although the same amount of DNA was added to the cells. The amount was almost proportional to the amount of DNA added and was the highest after addition of hexapodna, followed by pentapodna, tetrapodna, and tripodna, in this order. Similar results were obtained when the levels of TNF- $\alpha$ in the culture media were measured (data not shown). Figure 2B shows the normalized mean fluorescent intensity (MFI) values of DC2.4 cells after 8 h-incubation with Alexa Fluor 488-labeled ssDNA or polypodna preparations at $37^{\circ} \mathrm{C}$. The higher the pod number, the greater the MFI values of the cells. The MFI values of cells incubated with DNA samples at $4^{\circ} \mathrm{C}$ were much lower than those at $37^{\circ} \mathrm{C}$ (data not shown).

\section{Effect of inhibitors on IL-6 release and cellular uptake in DC2.4 cells}

Figure 2C shows the IL- 6 concentrations in the culture media of DC2.4 cells after addition of $\mathrm{CpG}$ hexapodna in the presence or absence of uptake inhibitors. All the inhibitors used significantly attenuated IL-6 release from the cells. Almost no IL-6 was released when chloroquine or cytochalasin B was added to the cells. When cells were treated with LPS, significant amounts of IL-6 were released, indicating that the cellular functions to release the cytokine were not impaired by the treatment. Figure 2D shows the MFI values of cells treated with each inhibitor followed by with Alexa Fluor 488-labeled hexapodna. Chloroquine had relatively little effect on the cellular uptake of Alexa Fluor 488-labeled hexapodna, whereas 
dansylcadaverine or cytochalasin B significantly reduced the uptake.

\section{IL-12 release from mouse splenic macrophages}

To investigate the responsiveness of primary immune cells to polypodna preparations, mouse splenic macrophages were used. Because splenic macrophages release higher amounts of IL-12 than IL-6, ${ }^{5}$ IL-12p40 was measured as an indicator of the activation of splenic macrophages. Figure 3A shows IL-12p40 concentrations in the culture media of splenic macrophages of C57/BL6 mice. The concentrations of IL-12p40 rose as the pod number increased. To confirm the importance of CpG motifs on cytokine release from splenic macrophages, DNA samples containing GpC sequences instead of $\mathrm{CpG}$ were added to splenic macrophages (Figure 3B). No significant amounts of IL-12p40 were released when DNA containing GpC sequences were added. DNA sensors other than TLR9 have also been reported to be involved in the DNA-mediated production of cytokines from immune cells. ${ }^{24}$ To determine if alternative sensors were involved in the responses, we examined cytokine release in splenic macrophages isolated from TLR9 ${ }^{-/-}$mice. As shown in Figure 3C, no significant release of IL-12p40 was observed from splenic macrophages of TLR9 ${ }^{-/-}$mice irrespective of the structure of $\mathrm{CpG}$ DNA.

\section{Uptake of polypodna in mouse splenic macrophages}

The differences in the release of IL-12p40 from splenic macrophages between wild-type and $\mathrm{TLR}^{-/-}$mice or between $\mathrm{CpG}$ and $\mathrm{GpC}$ samples might be due to the differences in the cellular uptake of DNA samples. Therefore, the uptake of Alexa Fluor 488-labeled hexapodna by splenic macrophages was examined (Figure 3D). There was no significant difference in the uptake of Alexa Fluor 488-labeled CpG hexapodna between wild-type and TLR9 ${ }^{-1-}$ macrophages. In addition, the uptake of Alexa Fluor 488-labeled GpC hexapodna by 
wild-type macrophages was comparable with that of Alexa Fluor 488-labeled CpG hexapodna.

\section{IL-6 release from mouse BMDCs}

Figure 4 shows the IL-6 concentrations in the culture media of BMDCs of C57/BL6 mice after addition of ssDNA or polypodna preparations. BMDCs released large amounts of IL-6 depending on the pod number. Again, no detectable IL-6 was released from BMDCs of TLR9 $^{-/-}$mice (data not shown).

\section{IL-12p40 production by polypodna after intradermal injection in mice}

ssDNA, CpG tripodna, or CpG hexapodna was injected into mice by intradermal injection to examine IL-12p40 production by these preparations in vivo. Figure 5 shows the time course of the serum IL-12p40 levels of mice after injection of saline (vehicle) or DNA samples. No significant increase in the IL-12p40 level was observed in mice receiving saline or ssDNA. In contrast, the level significantly increased in mice receiving $\mathrm{CpG}$ tripodna or $\mathrm{CpG}$ hexapodna.

\section{IFN- $\alpha$ release from human PBMCs}

Because the optimal CpG motif for human TLR9 is reportedly GTCGTT, which is not identical to the optimal murine sequence of GACGTT, ${ }^{25}$ polypodna preparations containing human CpG motifs were designed (Table 2). Figure 6A shows the PAGE analysis of ssDNA, tripodna, tetrapodna, pentapodna, and hexapodna. Every sample migrated as a single band on PAGE, indicating efficient formation of each polypodna preparation. Human PBMCs were incubated with ssDNA or polypodna preparations, and the release of IFN- $\alpha$ was measured. Figure 6B shows the IFN- $\alpha$ level in culture media of human PBMCs after addition of ssDNA or polypodna preparations. Compared with ssDNA, all polypodna preparations, except 
tripodna, induced significantly higher amounts of IFN- $\alpha$, although there were no significant differences among the polypodna preparations.

Inhibition of HCV replication by the conditioned media of PBMCs

An HCV subgenomic replicon system was used to examine whether polypodna-induced cytokine production is effective in inhibiting HCV replication in Huh-7 human hepatoma cells. Figure 6C shows the luciferase activity of LucNeo\#2 cells after incubation with or without addition of the conditioned medium of ssDNA- or hexapodna-treated PBMCs. The conditioned medium of the hexapodna-treated PBMCs reduced luciferase activity more effectively than that of the ssDNA-treated PBMCs. 


\section{Discussion}

There are many strategies to increase the immunostimulatory activity of CpG DNA, including the use of nuclease-resistant DNA analogues. ${ }^{9,10}$ We have reported that building up CpG DNA into branched DNA assemblies, such as polypodna and dendrimer-like DNA, is a novel and effective approach to increasing the immunostimulatory activity of CpG DNA. ${ }^{12,14,17,26}$ Using mouse macrophage-like RAW264.7 cells, we reported that highly branched DNA assemblies are more effective than conventional single-stranded CpG DNA in inducing cytokine production. We also demonstrated that tetrapodna preparations are more effective than ssDNA in inducing IL12p40 in mice after injection into the footpad. ${ }^{26}$ The present study has extended this finding by showing that other immune cells, i.e., mouse dendritic DC2.4 cells, mouse splenic macrophages, mouse BMDCs, and human PBMCs, respond to CpG polypodna more efficiently than to single-stranded CpG DNA. Similar results of pod number-dependent cytokine production were observed in mice. These results strongly suggest that assembly of CpG DNA into polypod-like or dendritic structures is a versatile and useful method by which to increase the potential of $\mathrm{CpG}$ DNA as a vaccine adjuvant without using chemically stabilized DNA analogues.

The detailed mechanism whereby polypodna preparations induce large amounts of cytokines has yet to be fully elucidated, but likely involves the efficient interaction of CpG DNA with its receptor, TLR9. One important factor contributing to the interaction is the cellular uptake of CpG DNA. We found that the cellular uptake of polypodna increased with increasing pod number in RAW264.7 cells ${ }^{17}$ and DC2.4 cells (Figure 2B). We also showed that the cellular uptake of hexapodna consisting of 240 nucleotides is higher than that of penta-, tetra- or tripodna of the same number of nucleotides. ${ }^{17}$ The significant inhibition of IL-6 production following treatment with the inhibitors (Figure 2C) used suggested that several mechanisms, including endosomal fusion and acidification, clathrin-mediated 
endocytosis, and phagocytosis, are involved in the uptake of polypodna by the cells. Taken together, these results advocate the hypothesis that DNA assemblies with a bulky structure have a high potential to interact with cells. This hypothesis is supported by experimental evidence that long ODNs are more efficiently taken up by RAW264.7 cells than short ones. ${ }^{27}$

It has been suggested that the cellular uptake of DNA is not mediated by the interaction of TLR9 and CpG DNA. ${ }^{28}$ Consistent with this, we found that the cellular uptake of $\mathrm{CpG}$ or $\mathrm{GpC}$ hexapodna in wild-type or $\mathrm{TLR} 9^{-/-}$splenic macrophages was similar (Figure 3D). Our previous study suggests that polypodna shares the uptake mechanism with single- or double-stranded DNA. ${ }^{17}$ Studies using phosphorothioate-stabilized CpG DNA concluded that long retention in endosomes explains the high ability of multimeric $\mathrm{CpG}$ DNA to produce large amounts of IFN- $\alpha$ from plasmacytoid dendritic cells (pDCs) compared with monomeric CpG DNA. ${ }^{29,30}$ Although the detailed mechanism governing the cellular uptake of polypodna remains to be determined, these results suggest that the structure-dependent increase in uptake leads to increased interaction of CpG DNA with TLR9 in endosomes due to its higher concentration and possibly longer retention time in these vesicles.

An important finding of the present study is that the immunostimulatory activity of CpG polypodna is solely dependent on TLR9 ligation. Splenic macrophages and BMDCs of $\mathrm{TLR}^{-/-}$mice released no significant amount of cytokines when treated with $\mathrm{CpG}$ polypodna preparations (Figure 3C and data not shown). When treated with imoquimod, a ligand for TLR7, TLR9 $^{-/}$splenic macrophages and BMDCs released large amounts of IL-12 and IL-6, respectively (data not shown), indicating that the cells have the ability to release cytokines. In addition, these results strongly suggest that TLR9, rather than other intracellular DNA sensors that recognize DNA irrespective of CpG motifs, ${ }^{24,31-33}$ is the major polypodna sensor. This was also supported by the almost complete inhibition of CpG hexapodna-induced IL-6 release from DC2.4 cells by chloroquine, an inhibitor of CpG-triggered cytokine release. ${ }^{34}$ 
Our previous in vivo study indicated that tetrapodna preparations are more effective in inducing IL-12p40 than ssDNA. The present study further confirmed this finding and clearly demonstrated that cytokine production is dependent on the pod number, as observed in experiments using cultured cells. These results provide experimental evidence that polypodna preparations with many pods are useful for in vivo applications compared with ones with fewer pods.

Human PBMCs consist of several types of cells, including TLR9-positive pDCs. It has been reported that pDCs release IFN- $\alpha$ upon recognition of CpG DNA by TLR9 ., 35 IFN- $\alpha$ activates many types of immune cells, including natural killer cells, ${ }^{36} \mathrm{DCs},{ }^{37} \mathrm{~T}$ cells, ${ }^{38}$ and B cells, ${ }^{39}$ so that the induction of IFN- $\alpha$ release would be useful for the initiation of an immune response. We found that polypodna induced significantly greater amounts of IFN- $\alpha$ secretion from human PBMCs than ssDNA (Figure 6B), although the pod number-dependent increase in human cells was less clear than in the murine counterparts. This might be explained by differences in experimental conditions, including the type of cells and cytokines measured. The PBMCs used would contain only $0.2-0.8 \%$ pDC and many more monocytes and T cells, according to the literature. ${ }^{35,40}$ With the exception of pDCs, human monocytes very weakly express TLR9. ${ }^{41,42}$ Therefore, cells other than pDCs, such as monocytes, might be involved in IFN- $\alpha$ release from human PBMCs.

The current standard therapy for $\mathrm{HCV}$ is a combination of pegylated IFN- $\alpha$ and ribavirin. ${ }^{43}$ Our preliminary study using the HCV subgenomic replicon system demonstrated that IFN- $\alpha$ treatment reduced the luciferase activity of the cells, but relatively high IFN- $\alpha$ concentrations (10 IU/mL, corresponding to about $2500 \mathrm{pg} / \mathrm{mL}$, or greater) were required for significant reduction in activity. Because the conditioned medium of human PBMCs containing about $50 \mathrm{pg} / \mathrm{mL}$ (20-fold diluted) inhibited the luciferase activity to less than $10 \%$ 
(Figure 6C), this clearly suggests that cytokines other than IFN- $\alpha$ are released from human PBMCs by addition of CpG hexapodna. Thus, hexapodna or other highly branched DNA assemblies, including dendrimer-like DNA, containing immunostimulatory sequences, such as CpG motifs, can be effective in inhibiting HCV replication.

In conclusion, we have demonstrated that building CpG DNA into polypod-like structures is a versatile and useful method by which to increase its uptake and cytokine production in many immune cell types, including human PBMCs, and in mice. Based on these findings, we propose that polypodna preparations are natural, biodegradable and highly potent vaccine adjuvants for the treatment of cancer and infectious diseases. 


\section{Figure Legends}

Figure 1. Sequence and planar representation of $\mathrm{CpG}$ polypodna for mouse cells. (A) $\mathrm{CpG}$ tripodna, (B) CpG tetrapodna, (C) $\mathrm{CpG}$ pentapodna, and (D) $\mathrm{CpG}$ hexapodna. Each ODN was identified with different colors.

Figure 2. IL-6 release and uptake of ssDNA or polypodna after addition to mouse dendritic DC2.4 cells. (A) DC2.4 cells were treated with ssDNA or polypodna at a final concentration of 1,2 or $4 \mu \mathrm{g} / \mathrm{mL}$ and incubated at $37^{\circ} \mathrm{C}$ for $24 \mathrm{~h}$. The concentrations of IL- 6 in culture media were measured by ELISA. Results are expressed as mean \pm SD of four wells. Data are representative of three independent experiments. $* \mathrm{P}<0.05$ compared with the ssDNA-treated group. (B) DC2.4 cells were treated with Alexa Fluor 488-labelled ssDNA or polypodna at a final concentration of $2 \mu \mathrm{g} / \mathrm{mL}$ and incubated at $37^{\circ} \mathrm{C}$ for $8 \mathrm{~h}$. The amounts of DNA associated with the cells were measured by flow cytometry, and the normalized MFI was calculated to compare the cellular uptake of DNA samples. Results are expressed as mean \pm $\mathrm{SD}$ of six wells. Data are representative of three independent experiments. ${ }^{*} \mathrm{P}<0.05$ compared with the ssDNA-treated group. (C) DC2.4 cells were incubated with none, $6.25 \mu \mathrm{M}$ chloroquine (CQ), $25 \mu \mathrm{M}$ monodansylcadaverine (MDC), or $5 \mu \mathrm{M}$ cytochalasin $\mathrm{B}(\mathrm{CyB})$ for $30 \mathrm{~min}$, then treated with hexapodna $(2 \mu \mathrm{g} / \mathrm{mL})$ or LPS $(25 \mathrm{ng} / \mathrm{mL})$ and incubated at $37^{\circ} \mathrm{C}$ for 24 h. The concentrations of IL-6 in culture media were measured by ELISA. Results are expressed as mean \pm SD of four wells. Data are representative of two independent experiments. ${ }^{*} \mathrm{P}<0.05$ compared with the untreated (control) groups. (D) DC2.4 cells were incubated with none, $6.25 \mu \mathrm{M}$ chloroquine (CQ), $25 \mu \mathrm{M}$ monodansylcadaverine (MDC), or 5 $\mu \mathrm{M}$ cytochalasin $\mathrm{B}(\mathrm{CyB})$ for $30 \mathrm{~min}$, then treated with hexapodna $(2 \mu \mathrm{g} / \mathrm{mL})$ and incubated at $37^{\circ} \mathrm{C}$ for $8 \mathrm{~h}$. The amounts of DNA associated with the cells were measured by flow 
cytometry. Results are expressed as the \% of control (the none group; MFI, 176.2) \pm SD of four wells. Data are representative of two independent experiments. $* \mathrm{P}<0.05$ compared with the none (control) group.

Figure 3. IL-12p40 release and uptake of ssDNA or polypodna after addition to mouse splenic macrophages. (A-C) Splenic macrophages from (A, B) wild-type C57/BL6 mice or (C) $\mathrm{TLR}^{-/-}$mice were treated with ssDNA or polypodna containing (A, C) $\mathrm{CpG}$ or (B) GpC motifs at a final concentration of (A) 1,2 or $4 \mu \mathrm{g} / \mathrm{mL}$ or $(\mathrm{B}, \mathrm{C}) 2 \mu \mathrm{g} / \mathrm{mL}$ and incubated at $37^{\circ} \mathrm{C}$ for $24 \mathrm{~h}$. The concentrations of IL-12p40 in culture media were measured by ELISA. Results are expressed as mean $\pm \mathrm{SD}$ of four wells. Data are representative of three independent experiments. ${ }^{*} \mathrm{P}<0.05$ compared with $(\mathrm{A})$ the ssDNA-treated group or $(\mathrm{B}, \mathrm{C})$ the medium-treated group. (D) Splenic macrophages from wild-type or TLR9 ${ }^{-/-}$mice were treated by Alexa Fluor 488-labelled hexapodna containing $\mathrm{CpG}$ or $\mathrm{GpC}$ motifs at a final concentration of $2 \mu \mathrm{g} / \mathrm{mL}$ and incubated at $37^{\circ} \mathrm{C}$ for $4 \mathrm{~h}$. The amounts of DNA associated with cells were measured by flow cytometry. Results are expressed as mean \pm SD of three wells. Data are representative of two independent experiments.

Figure 4. IL-6 release after addition of ssDNA or polypodna to mouse BMDCs. BMDCs were treated with ssDNA or polypodna at a final concentration of $2 \mu \mathrm{g} / \mathrm{mL}$ and incubated at $37^{\circ} \mathrm{C}$ for $24 \mathrm{~h}$. The concentrations of IL-6 in culture media were measured by ELISA. Results are expressed as mean \pm SD of three wells. Data are representative of three independent experiments. $* \mathrm{P}<0.05$ compared with the ssDNA-treated group.

Figure 5. Serum IL-12p40 concentration after intradermal injection of ssDNA or polypodna 
into mice. Mice received an injection of ssDNA or polypodna at a dose of $100 \mu \mathrm{g} / \mathrm{mouse}$, and blood was collected from the tail vein at indicated periods of time after injection. The concentrations of IL-12p40 in serum were measured by ELISA. Results are expressed as mean \pm SD of six mice. Data are representative of two independent experiments. $* \mathrm{P}<0.05$ compared with the ssDNA-treated group.

Figure 6. IFN- $\alpha$ release from human PBMCs and inhibition of HCV replication in LucNeo\#2 cells after addition of ssDNA or polypodna to human PBMCs. (A) PAGE analysis of polypodna preparations containing human $\mathrm{CpG}$ motifs. Each sample was run on a $6 \%$ polyacrylamide gel at $200 \mathrm{~V}$ for $20 \mathrm{~min}$. Lane 1, 100-bp DNA ladder; lane 2, ssDNA; lane 3, tripodna; lane 4, tetrapodna; lane 5, pentapodna; lane 6, hexapodna. (B) PBMCs were treated with ssDNA or polypodna at a final concentration of $2 \mu \mathrm{g} / \mathrm{mL}$ and incubated at $37^{\circ} \mathrm{C}$ for $24 \mathrm{~h}$. The concentrations of IFN- $\alpha$ in culture media were measured by ELISA. Results are expressed as mean \pm SD of five wells. Data are representative of four independent experiments. ${ }^{*} \mathrm{P}<0.05$ compared with the ssDNA-treated group. (C) PBMCs were incubated with ssDNA or polypodna as described above. Then, the conditioned medium was diluted 20-fold, added to LucNeo\#2 cells, and the cells were incubated at $37^{\circ} \mathrm{C}$ for $24 \mathrm{~h}$. Finally, the luciferase activity of the cell lysates was measured as an indicator of HCV replication. Results are expressed as mean \pm SD of five wells. Data are representative of three independent experiments. $* \mathrm{P}<0.05$ compared with the ssDNA-treated group. 
Table 1. The sequences of ODNs used for polypodna preparations for mouse cells.

\begin{tabular}{|c|c|}
\hline ODN & Sequence $\left(5^{\prime} \rightarrow 3^{\prime}\right)$ \\
\hline mTri-1 & TCGTCAACGTCTGTGCTCTCACGTTGACGCTGTCGA \\
\hline mTri-2, mTet-2, mPen-2, mHex-2 & TCGACAGCGTCAACGTGAAACGTGAAGCGTCTGCGA \\
\hline mTri-3, mPen-3, mHex-3 & TCGCAGACGCTTCACGTTGAGCACAGACGTTGACGA \\
\hline mTet-1, mPen-1, mHex-1 & TCGCTGACGTTGCAGACATCACGTTGACGCTGTCGA \\
\hline mTet-3 & TCGCAGACGCTTCACGTTGCAGACAGACGTTGACGA \\
\hline mTet-4 & TCGTCAACGTCTGTCTGCTGTCTGCAACGTCAGCGA \\
\hline mPen-4, mHex-4 & TCGTCAACGTCTGTGCTCGCAGCGTCTTAACGTCGA \\
\hline mPen-5 & TCGACGTTAAGACGCTGCTGTCTGCAACGTCAGCGA \\
\hline mHex-5 & TCGACGTTAAGACGCTGCAGACGTTCAGGACTACGA \\
\hline mHex-6 & TCGTAGTCCTGAACGTCTTGTCTGCAACGTCAGCGA \\
\hline mGCTri-1 & TGCTCAAGCTCTGTGCTC TCAGCTTGAGCCTGTGCA \\
\hline mGCTri-2, mGCTet-2, mGCPen-2, & TGCACAGGCTCAAGCTGA AAGCTGAAGGCTCTGGCA \\
\hline \multicolumn{2}{|l|}{ mGCHex-2 } \\
\hline mGCTri-3, mGCPen-3, mGCHex-3 & TGCCAGAGCCTTCAGCTT GAGCACAGAGCTTGAGCA \\
\hline mGCTet-1, mGCPen-1, mGCHex-1 & TGCCTGAGCTTGCAGACA TCAGCTTGAGCCTGTGCA \\
\hline mGCTet-3 & TGCCAGAGCCTTCAGCTT GCAGACAGAGCTTGAGCA \\
\hline mGCTet-4 & TGCTCAAGCTCTGTCTGC TGTCTGCAAGCTCAGGCA \\
\hline mGCPen-4, mGCHex-4 & TGCTCAAGCTCTGTGCTC GCAGGCTCTTAAGCTGCA \\
\hline mGCPen-5 & TGCAGCTTAAGAGCCTGC TGTCTGCAAGCTCAGGCA \\
\hline mGCHex-5 & TGCAGCTTAAGAGCCTGC AGAGCTTCAGGACTAGCA \\
\hline mGCHex-6 & TGCTAGTCCTGAAGCTCT TGTCTGCAAGCTCAGGCA \\
\hline mGCHex-6 & TGCTAGTCCTGAAGCTCT TGTCTGCAAGCTCAGGCA \\
\hline
\end{tabular}

All ODNs have a phosphodiester backbone. 
Table 2. The sequences of ODNs used for polypodna preparations for human cells.

\begin{tabular}{ll}
\hline ODN & Sequence $\left(5^{\prime} \rightarrow 3^{\prime}\right)$ \\
\hline hTri-1, hTet-1, hPen-1, hHex-1 & TCGTCGTTCCGTCGTTACACTGCTCTGGCGGTCGTT \\
hTri-2, hTet-2, hPen-2, hHex-2 & AACGACCGCCAGAGCAGTCGTGTCGTACTACGACGA \\
hTri-3 & TCGTCGTAGTACGACACGGTAACGACGGAACGACGA \\
hTet-3, hPen-3, hHex-3 & TCGTCGTAGTACGACACGGTCGTAAGCCTGGTCGTA \\
hTet-4 & TACGACCAGGCTTACGACGTAACGACGGAACGACGA \\
hPen-4, hHex-4 & TACGACCAGGCTTACGACAGTCTAGCTGATCGACGA \\
hPen-5 & TCGTCGATCAGCTAGACTGTAACGACGGAACGACGA \\
hHex-5 & TCGTCGATCAGCTAGACTGCTGTCGATGCCAACGAC \\
hHex-6 & GTCGTTGGCATCGACAGCGTAACGACGGAACGACGA \\
hGCTri-1, hGCTet-1, hGCPen-1, & TGCTGCTTGCCTGCTTACACTGCTCTGGCGGTGCTT \\
hGCHex-1 & \\
hGCTri-2, hGCTet-2, hGCPen-2, & AAGCACCGCCAGAGCAGTGCTGTGCTACTAGCAGCA \\
hGCHex-2 & \\
hGCTri-3 & \\
hGCTet-3, hGCPen-3, hGCHex-3 & TGCTGCTAGTAGCACAGCGTGCTAAGCCTGGTGCTA \\
hGCTet-4 & TAGCACCAGGCTTAGCACGTAAGCAGGCAAGCAGCA \\
hGCPen-4, hGCHex-4 & TGCACACACAGGCTTAGCACAGTCTAGCTGATGCAGCA \\
\hline
\end{tabular}

All ODNs have a phosphodiester backbone. 


\section{Figure 1}

(A)

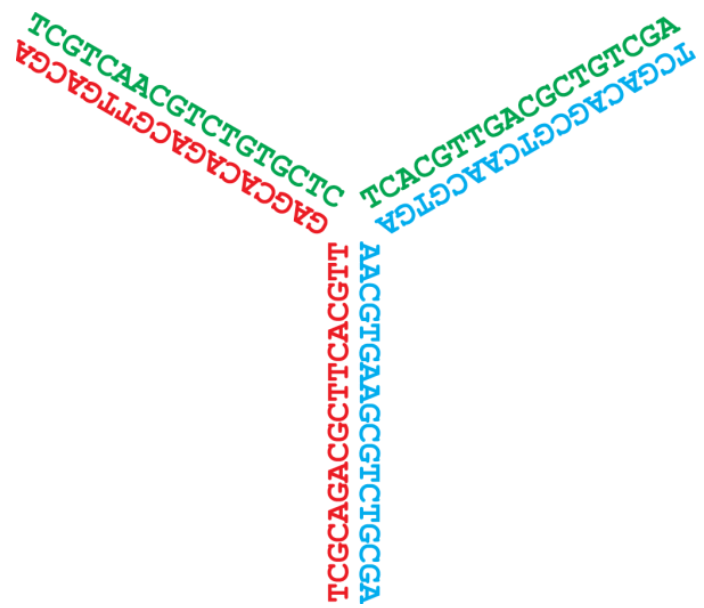

(C)

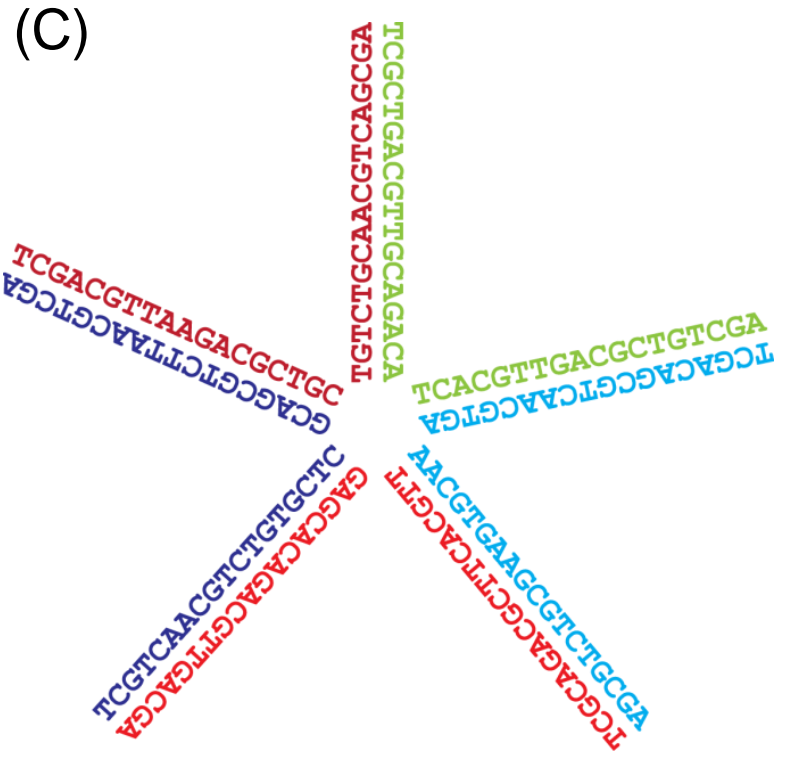

(B)

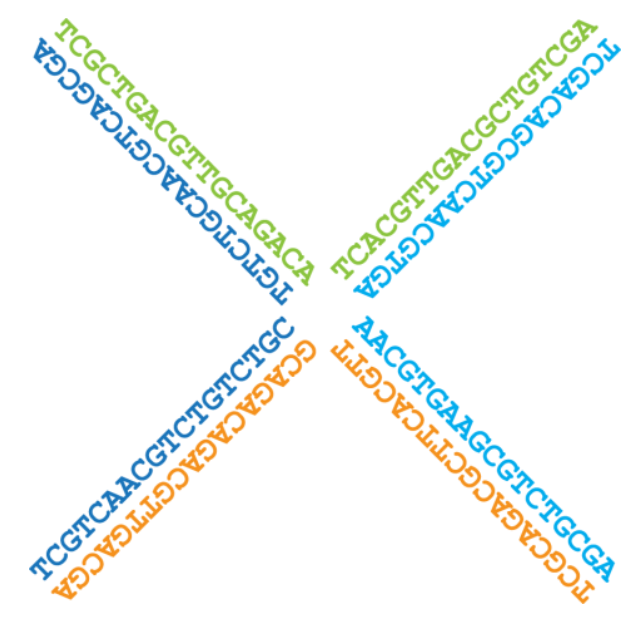

(D)

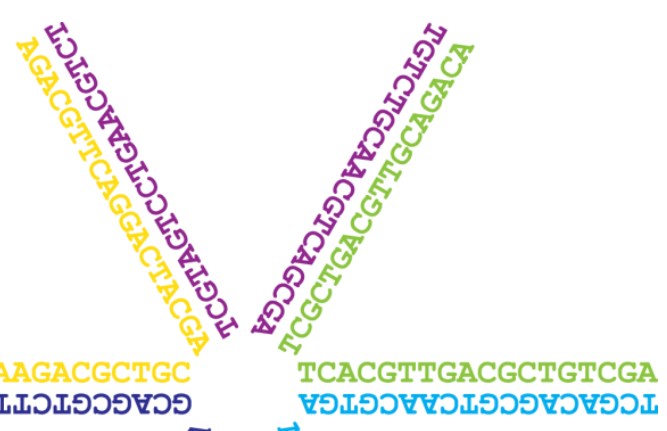

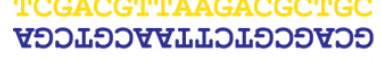

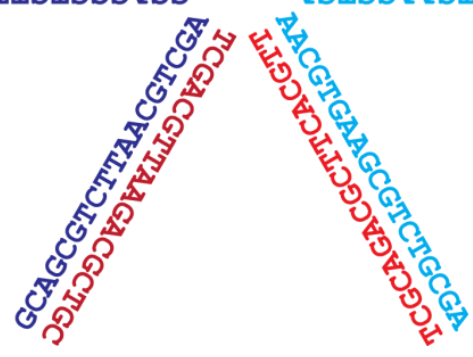




\section{Figure 2 (DC2.4)}

\section{(A)}

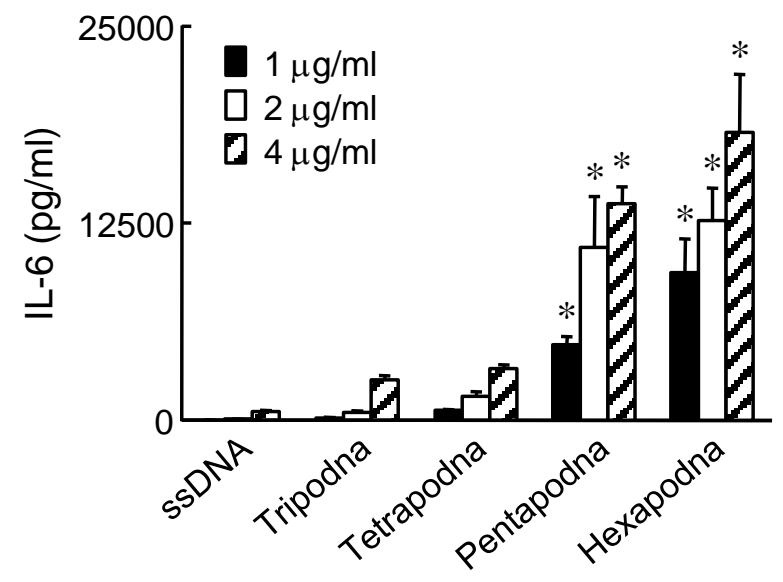

(B)

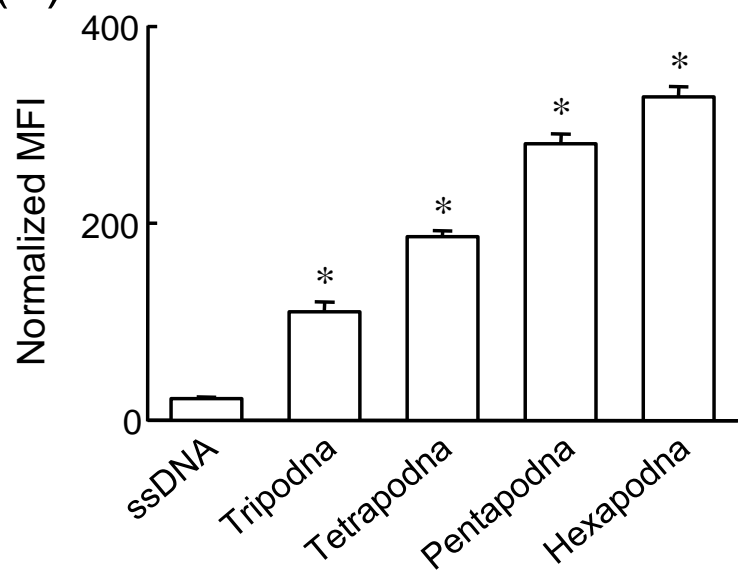

(C)

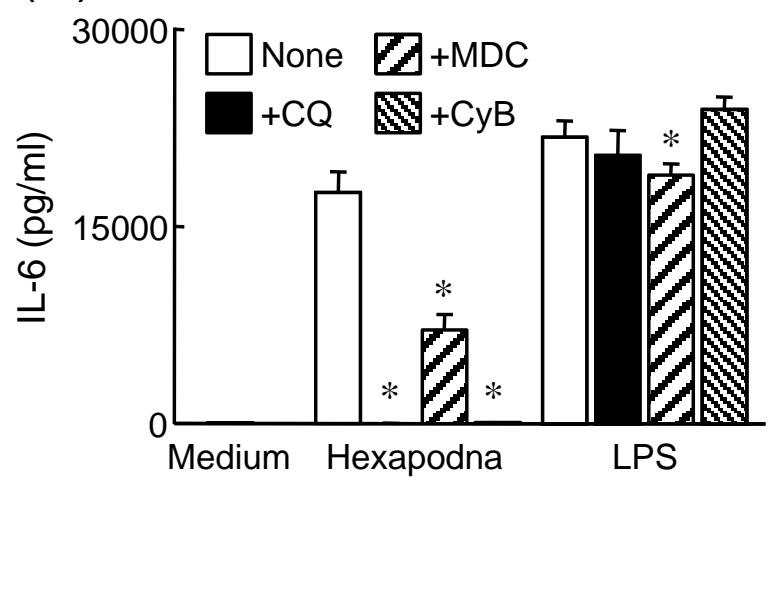

(D)

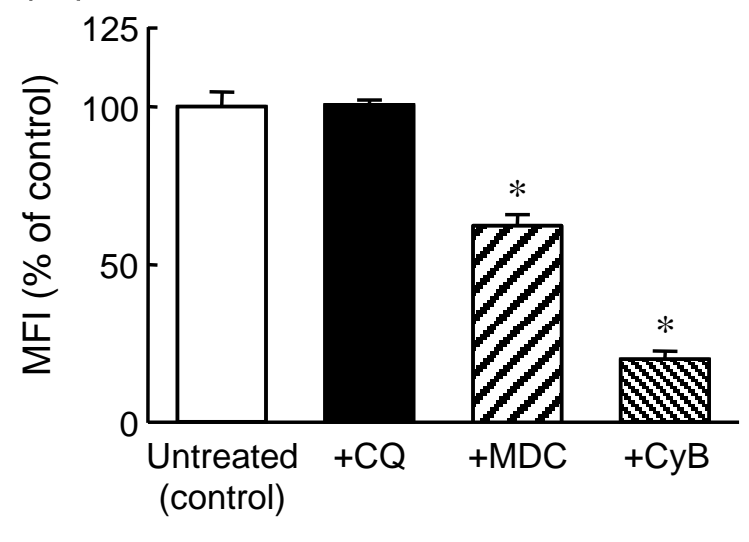


京都大学
KYOTO UNIVEASTY

A Self-archived copy in
Kyoto University Research Information Repository

\section{Figure 3 (Spl Mac) NEW}

(A)

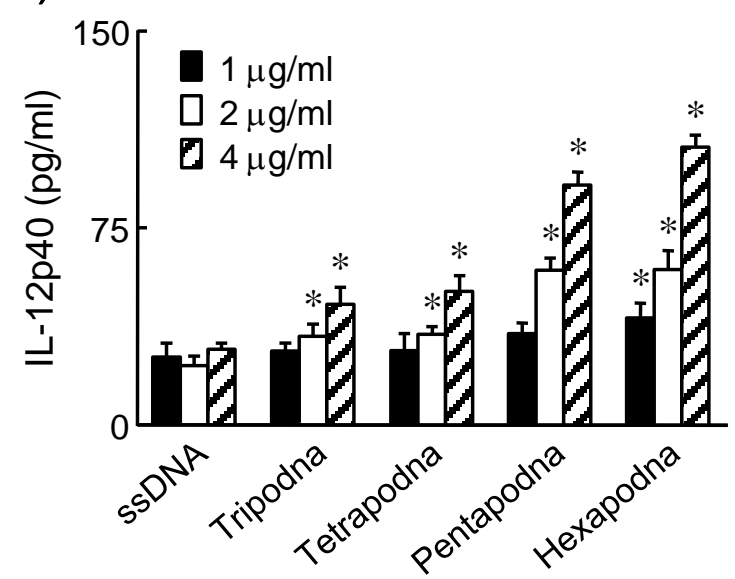

(B)

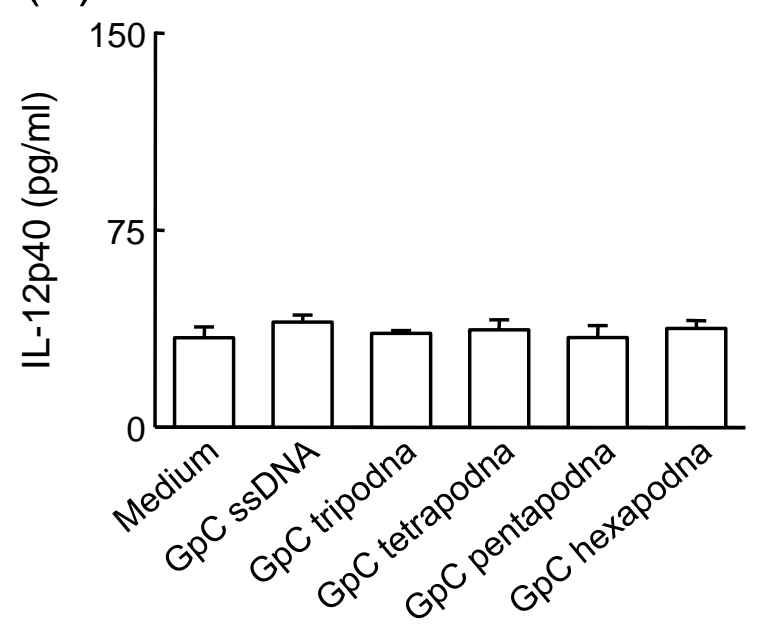

(C)

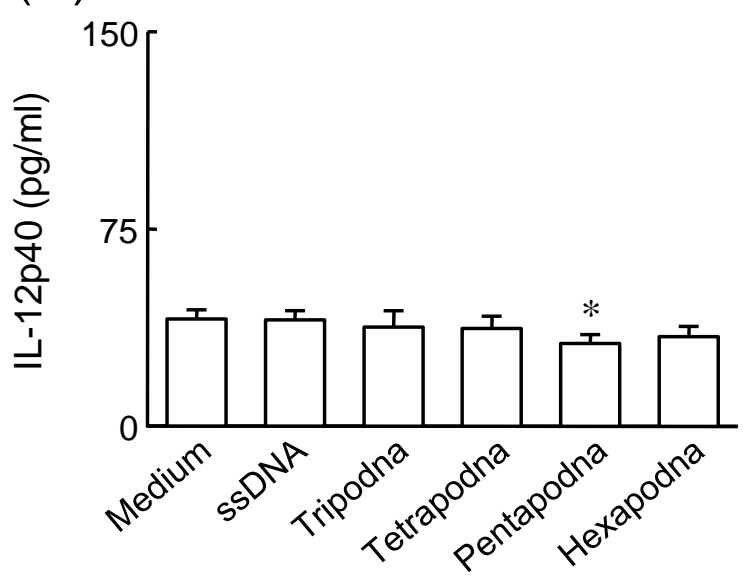

(D)

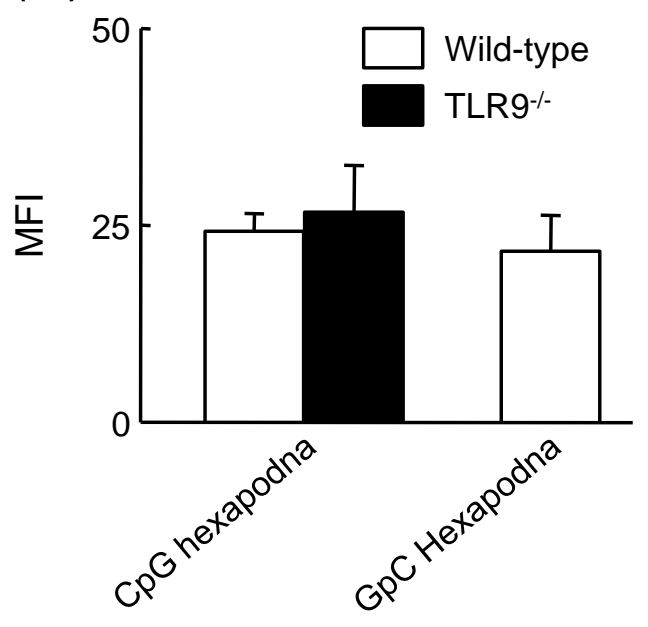




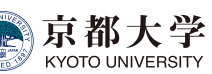

\section{Figure 4 (BMDC)}

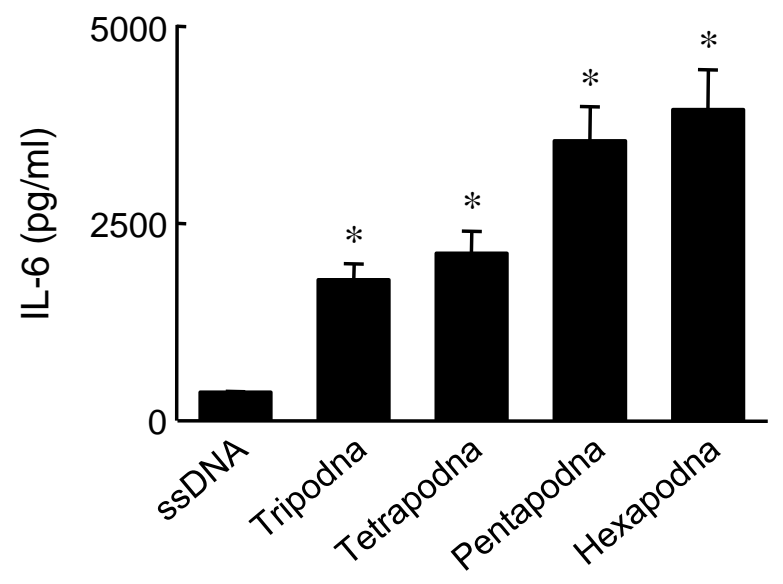




\section{Figure 5 (Mouse, i.d.)}

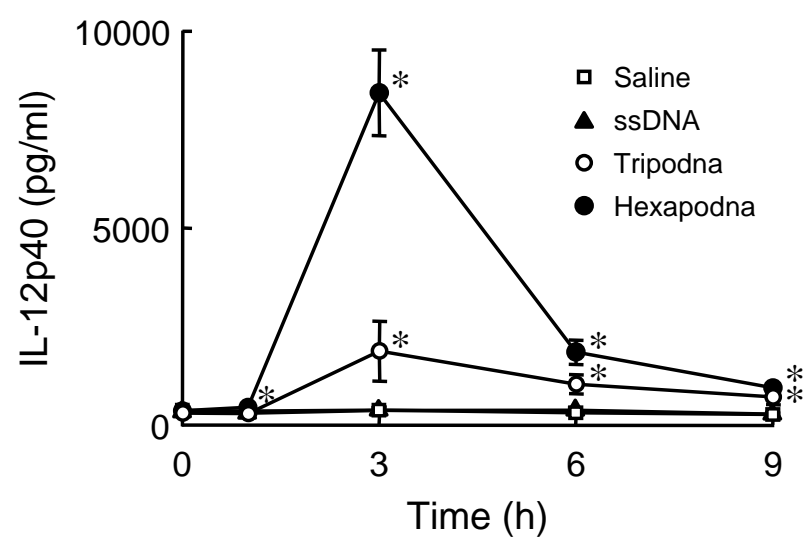




\section{Figure 6 (PBMC)}

(A)

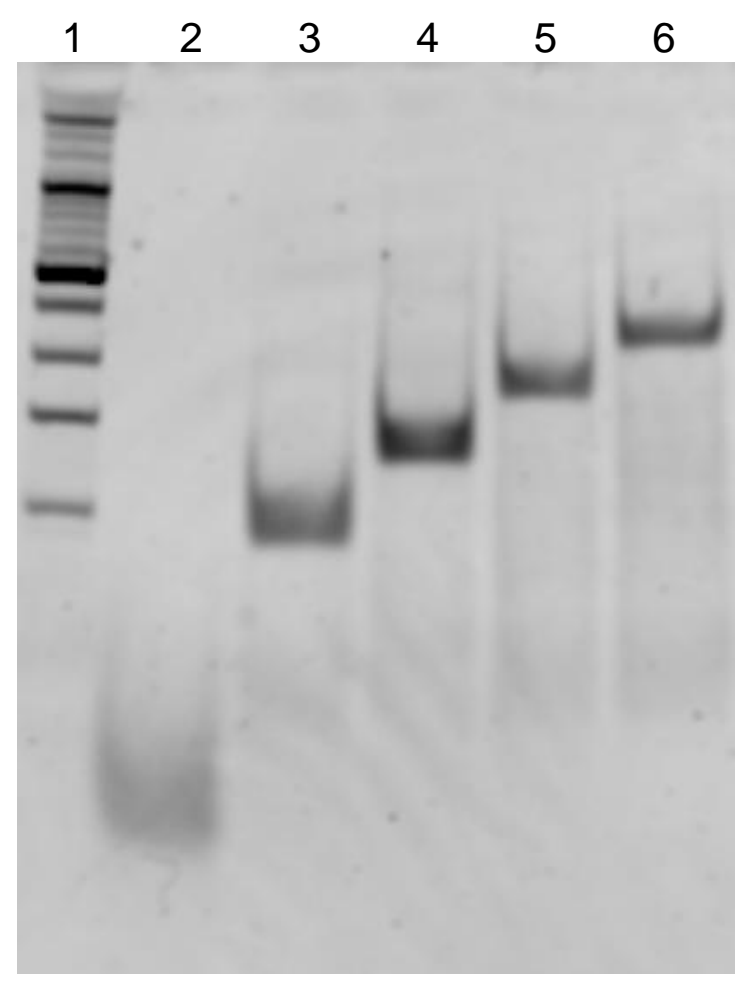

(B)
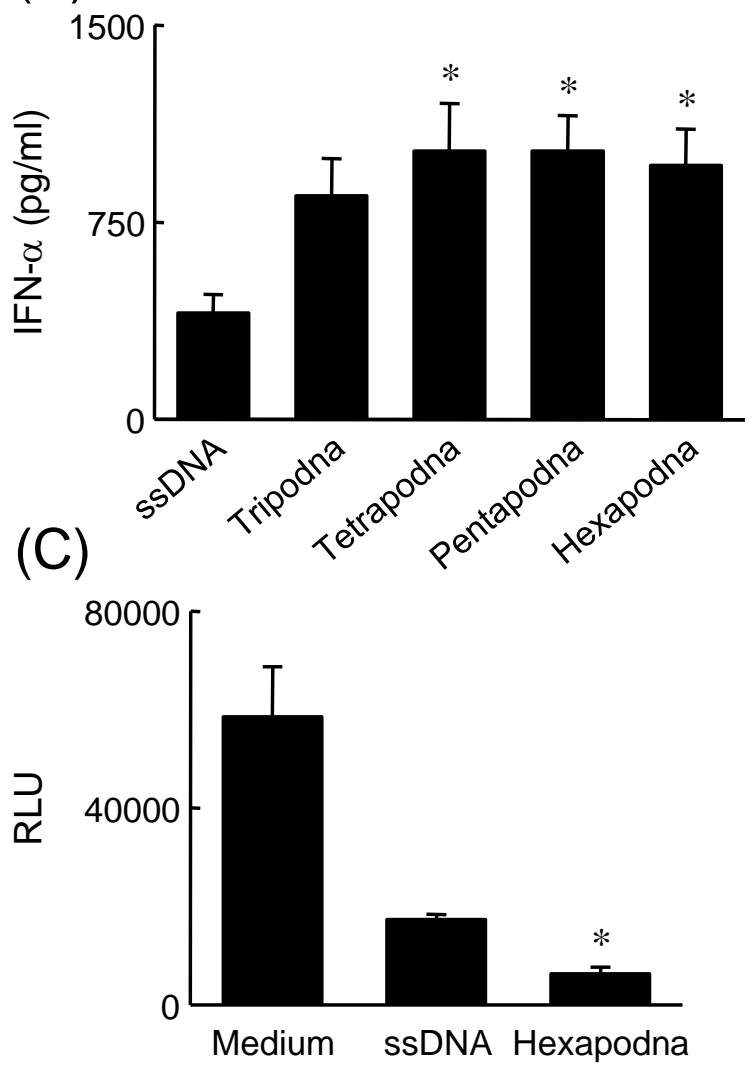